\title{
Efficient Analysis of Monosaccharides and Oligosaccharides from Hydrolyzed Hemicellulose of Spartina anglica
}

\author{
Rusheng Xie, ${ }^{a}$ Meng Li,${ }^{b}$ Suixiang Ma, ${ }^{a}$ Jian Liu, ${ }^{a}$ and Minnan Long a,b,* \\ Spartina anglica, a plant that controls coastal erosion, is widely \\ distributed throughout the world and is rich in cellulose, hemicellulose, \\ and lignin. The hemicellulose from Spartina anglica can be extracted and \\ hydrolyzed into monosaccharides and xylooligosaccharides under acid \\ or enzymatic digestion conditions. In this study, an effective PMP(1- \\ phenyl-3-methyl-5-pyrazolone)-derivatized HPLC (High performance \\ liquid chromatography) method was developed for monitoring \\ monosaccharides and xylooligosaccharides of Spartina anglica. With \\ phosphate buffer $(0.04 \mathrm{M}, \mathrm{pH} 8.06)$ as mobile phase $A$, and acetonitrile \\ as mobile phase $B$, in which the elution gradient was set as $A: B / 79: 21$, \\ the monosaccharides (glucose, xylose and arabinose) and \\ xylooligosaccharides (xylobiose, xylotriose, xylotetraose, xylopentaose, \\ xylohexaose) could be separated completely using the C18 column. This \\ provides an economical, rapid, and efficient method for process \\ monitoring in the bioconversion of Spartina anglica.
}

Keywords: Spartina anglica; Hemicellulose; Xylooligosaccharides; PMP derivatization; HPLC

Contact information: a: Fujian Vocational College of Bioengineering, Fuzhou 350002, Fujian, China;

b: College of Energy, Xiamen University, Xiamen 361102, Fujian, China;

*Corresponding author: Longmn@xmu.edu.cn

\section{INTRODUCTION}

Spartina anglica was introduced to China in 1980s for coastal erosion control. This plant is resistant to salt, water, and high-pH soil. To prevent the expansion of tidal flats, Fujian province introduced $S$. anglica from abroad in the 1980s. It has played a vital role in creating land with silt, thus protecting river banks. However, rapid propagation and tenacious vitality make it grow out of control in the coastal beaches, which results in severe destruction of original ecosystems. Moreover, aquatic plants and others have difficulty surviving in this area. Although $S$. anglica can be converted into fuel and other high-value products, an analysis method of monosaccharide and oligosaccharides has not yet been established.

Spartina anglica is rich in cellulose, hemicellulose, and lignin. Traditionally, natural cellulose raw material treatment is mainly for the utilization of cellulose, but the hemicellulose and lignin have not been fully utilized, resulting in great waste of resources and environmental pollution. Cellulose, hemicellulose, and lignin are polymers with complex spatial structures. In natural cellulose materials, they aggregate into complex supramolecular compounds. Most lignin exists in intercellular layers and forms a solid binding net framework with hemicellulose, which provides a protective layer on cellulose. Therefore, it is quite difficult to separate the three components completely for their utilization in industry.

As a homogeneous glycan, cellulose is connected by beta-1,4 glycosidic bond with 
D-glucose, in which the main hydrolysates are cellobiose and glucose (Lin and Tanaka 2006). Unlike cellulose, hemicelluloses exists as heterogeneous glycan with more complex components. Hemicellulose from different biomass sources has different components, but the major carbohydrates include D-glucose, D-xylose, L-Arabia sugar, D-mannose, Dgalactose, L-rhamnose, 4-O-methyl glucuronic acid, and galactose uronic acid (Peng et al. 2012). Hemicellulose plays an important role in the process of lignocellulose hydrolysis (Zhang et al. 2012a, b). Xylan and arabino-glycan of lignocellulose can be transformed into functional sugar such as xylooligosaccharides and arabinose by acid or enzymatic hydrolysis (Lavarack et al. 2002). Hence, the production of xylose or xylooligosaccharides from $S$. anglica has a huge market prospect. HPLC, gas chromatography (GC), and ion chromatography are common methods for the analysis and determination of carbohydrates (Arfelli and Sartini 2014). However, there is still no well-established method to analyze the complex lignocellulosic biomass hydrolyzed products, especially the xylooligosaccharides.

Saccharides lack ultraviolet absorption and are usually detected by HPLC with a refractive index differential detector (RID). HPLC-RID has low sensitivity to saccharides and poor separation effect on xylooligosaccharides (Brienzo et al. 2010; Thamyres et al. 2020). Because carbohydrates are difficult to volatilize and detect by GC only using indirect methods, carbohydrates generally are derivatized into volatile compounds and then detected. However, derivatization is complicated and only suitable for monosaccharide detection, and there have not been many reports on the detection of oligosaccharide. The compound 1-phenyl-3-methyl-5-pyrazolone (PMP) increases the ionization degree of sugar and detection sensitivity (Honda et al. 1989). Therefore, PMP pre-column derivatization HPLC has been widely applied to monosaccharide analysis (You et al. 2009). To date, this method has not been applied to simultaneous analysis of monosaccharides and oligosaccharides in Spartina biomass.

In the previous reports, residual PMP reagent after derivatization reaction was generally degraded by adopting multiple extraction and drying. The PMP derivatives were easily lost, and the operation was complicated, which increased the chance of system error. In this paper, under the optimized conditions, since PMP is eluted first and the drying step can be omitted, the above disadvantages were overcome by directly analyzing the extracted sample. The newly established method yielded monosaccharides and oligosaccharides from $S$. anglica hydrolysis from a simplified process that improved separation efficiency. In this paper, a PMP derivatization HPLC method was established to analyze monosaccharides and oligosaccharides from hydrolysate of Spartina anglica hemicellulose.

\section{EXPERIMENTAL}

\section{Extraction of Hemicellulose}

Spartina anglica raw material was harvested from the seashore of Ningde, Fujian Province, China. It was dried and crushed into powder. The powder was soaked with $2 \%$ $\mathrm{NaOH}$ and $1 \% \mathrm{H}_{2} \mathrm{O}_{2}$ for $2 \mathrm{~h}$ at $60{ }^{\circ} \mathrm{C}$, then filtered and extracted with $2 \% \mathrm{NaOH}$ for $2 \mathrm{~h}$ at $100{ }^{\circ} \mathrm{C}$. The liquid from both steps was combined, concentrated, and precipitated with 2 volumes of $95 \%$ ethanol. The sediment was washed twice with $70 \%$ ethanol, air-dried, and used as the crude hemicellulose (Thamyres et al. 2020). The yield of crude hemicellulose was obtained as $22.5 \%$. 


\section{Hemicellulose Hydrolysis}

Two grams of crude hemicellulose powder were resuspended in $100 \mathrm{~mL}$ citrate buffer (50 mM, pH 5.0) containing $0.02 \%$ azide. The dosage of xylanase (Genuo, China) preparation was $200 \mathrm{IU} / \mathrm{g}$ dry matter. One unit (1 U) enzyme equals the amount of enzyme degradation and release $1 \mu \mathrm{mol}$ reducing sugar per min from xylan solution $(5 \mathrm{mg} / \mathrm{mL})$ at $37{ }^{\circ} \mathrm{C}$ and $\mathrm{pH}$ 5.5. Hydrolysis was performed in flasks in a water bath rolling at $50{ }^{\circ} \mathrm{C}$ for $0.5 \mathrm{~h}$. Hydrolysis samples were boiled for $10 \mathrm{~min}$, centrifuged at $8000 \mathrm{rpm}$ for $5 \mathrm{~min}$, filtered, and stored at $4{ }^{\circ} \mathrm{C}$ for further analysis.

\section{Purity and Structural Characterization of Hemicellulose}

Hemicellulose $(0.3 \mathrm{~g})$ was added into $4 \% \mathrm{H}_{2} \mathrm{SO}_{4}$ and incubated at $120{ }^{\circ} \mathrm{C}$ for $1 \mathrm{~h}$. The solution was neutralized with calcium carbonate and centrifuged at $12000 \mathrm{rpm}$ for 5 min. The supernatant was analyzed by HPLC (2695-2996, Waters, Milford, MA, USA) with Aminex (HPX-87H, BioRad, Hercules, CA, USA). The assay conditions were as follows: mobile phase of $5 \mathrm{mM} \mathrm{H}_{2} \mathrm{SO}_{4}$, at a speed of $0.6 \mathrm{~mL} / \mathrm{min}$, sample injection volume of $20 \mu \mathrm{L}$, column temperature at $35^{\circ} \mathrm{C}$, RID as monitor, and runtime for $15 \mathrm{~min}$.

$\mathrm{KBr}$ powder was dried to constant weight in electro-thermostatic oven (DHG9123A, Shanghai Jing Hong laboratory equipment Co., Ltd., Shanghai, China). Samples and $\mathrm{KBr}$ were added into agate mortar with mass ratio of 1:100, which were ground at the infrared lamp. Milled powder was pressed to slice in the tablet compression machine. The slice was measured in the infrared spectrometer (Nicolet 330, ThermoFisher Scientific, Waltham, MA, USA) at scanning wavelength of 4000 to $400 \mathrm{~cm}^{-1}$.

\section{Preparation of PMP Derivatives of Saccharides}

Monosaccharides derivatized by PMP have strong absorption peak at $245 \mathrm{~nm}$, and the structure of derivatives are so stable that they have little interference for analysis (Honda et al. 1989; Zhang et al. 2003). The mechanism of PMP derivatization is shown in Fig. 1, with the reaction of PMP and glucose as an example.
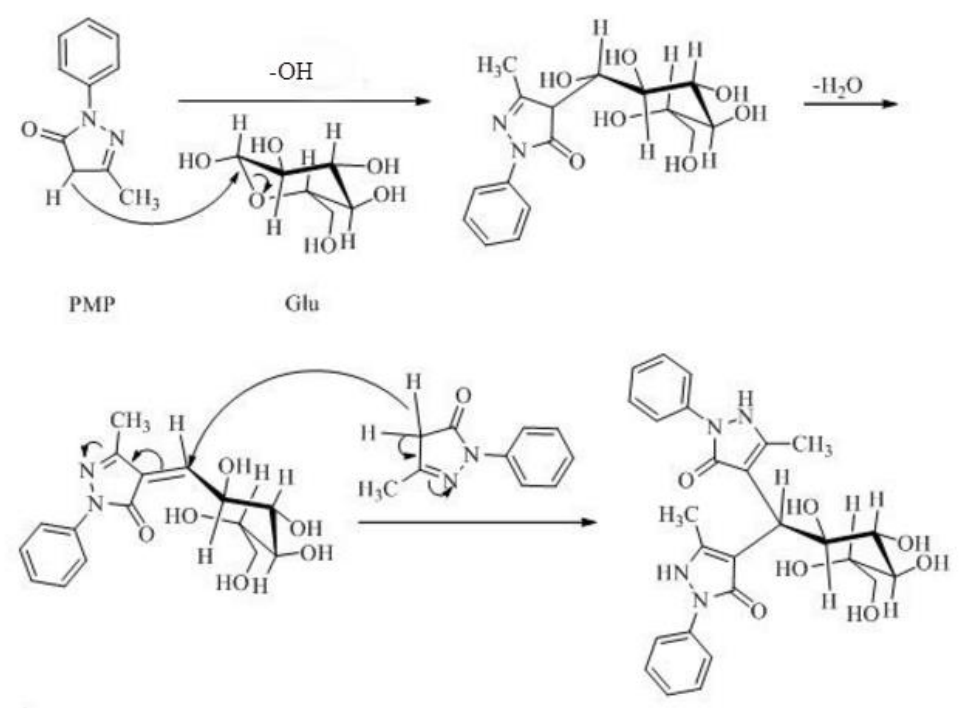

Fig. 1. Process and mechanism of PMP derivatization 
Saccharides standard solution (Purity $>90 \%$, Megazyme, Bray, Ireland) or hydrolysate of Spartina anglica hemicelluloses $(100 \mu \mathrm{L})$ were added into $\mathrm{NaOH}$ solution, and mixed with $100 \mu \mathrm{L} 0.5 \mathrm{M}$ PMP (AR, Acros Organics, Geel, Belgium)-methanol solution. The reaction was sustained for $30 \mathrm{~min}$ at $70^{\circ} \mathrm{C}$. The solution was cooled to room temperature, and $100 \mu \mathrm{L}$ of $0.3 \mathrm{M} \mathrm{HCl}$ was added to neutralize it. The extraction was performed with $1 \mathrm{~mL}$ of $\mathrm{CHCl}_{3}$, and organic solvent was removed after vortex oscillation. This process was repeated three times, and the extract was centrifuged at 12,000 rpm for 5 min. The supernatant was diluted to a certain ratio and tested with $0.22 \mu \mathrm{m}$ membrane filter.

\section{Optimization of Separation Condition for HPLC}

HPLC separation conditions were previously described (Li et al. 2013). The testing conditions were as follows: $\mathrm{C}_{18}$ chromatographic column (Shiseido, Tokyo, Japan), UV detection wavelength at $245 \mathrm{~nm}$, mobile phase A of phosphate buffer $(0.04 \mathrm{M}, \mathrm{pH} 8.00)$, mobile phase $\mathrm{B}$ of acetonitrile, and gradient elution $(\mathrm{A}: \mathrm{B}=79: 21)$. The velocity was adjusted from $0.5 \mathrm{~mL} / \mathrm{min}$ to $0.4 \mathrm{~mL} / \mathrm{min}$ for better separation.

\section{RESULTS AND DISCUSSION}

\section{Purity and Structural Characterization of Hemicellulose}

Hemicellulose is a complex structure with the main chain and side chain. The main chain of hemicellulose consists of D-xylose. The side chain of hemicellulose usually consists of L-arabinose, D-glucose, D-mannose, L-rhamnose, 4-O-methylglucuronic acid, and galactose uronic acid. After the crude hemicellulose $(0.3 \mathrm{~g})$ was completely hydrolyzed by $\mathrm{H}_{2} \mathrm{SO}_{4}, 0.01473 \mathrm{~g}$ glucose, $0.1928 \mathrm{~g}$ xylose, and $0.01974 \mathrm{~g}$ arabinose were obtained according to calculation based on the standard work curve (Fig.2). The results showed that arabinose and glucose were the main sugar groups of side chains. The mass content of hemicellulose reached $62.35 \%$, which indicates a high purity of hemicellulose and possibility of application in the industry.

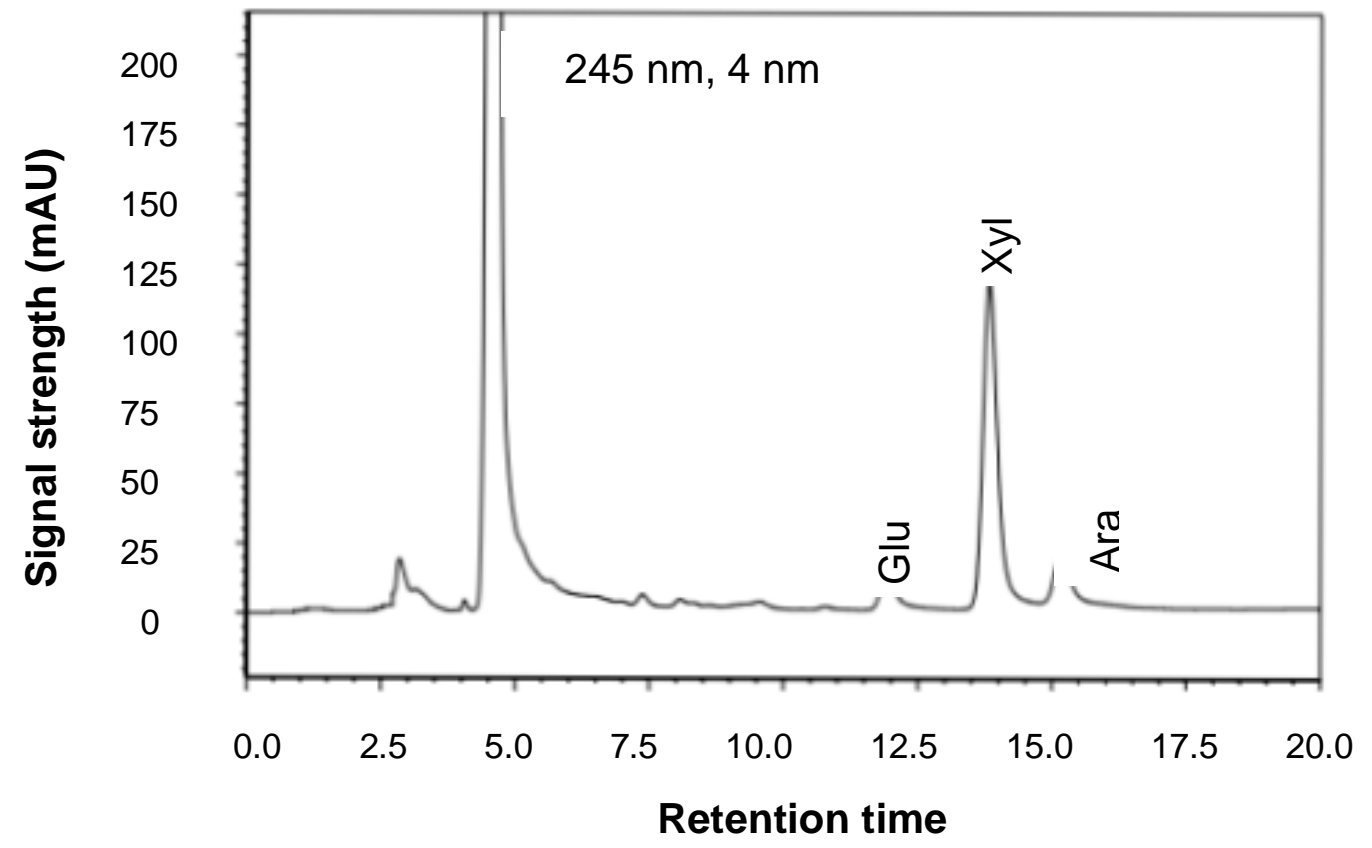

Fig. 2. HPLC analysis of hemicellulose hydrolysate from Spartina anglica 


\section{FT-IR Analysis}

FT-IR is a common technique used to determine the structure of polysaccharides. The absorption region changes as chemical groups interact at different molecular levels. Hemicellulose is a polysaccharide with a complex infrared absorption peak, and its typical peaks are observed at 1200 to $900 \mathrm{~cm}^{-1}$. Infrared spectrum results showed the hemicellulose extracted from Spartina anglica had a typical xylan structure (Fig. 3). Cao et al. (2015) assumed that the bands at $1504 \mathrm{~cm}^{-1}, 1240 \mathrm{~cm}^{-1}$, and $1040 \mathrm{~cm}^{-1}$ showed the relative concentration in lignin, cellulose, and hemicellulose, respectively. The strong absorption peak at $1040 \mathrm{~cm}^{-1}$ indicated the presence of hemicellulose, as attributed to the $\mathrm{C}-\mathrm{O}$ bond stretching vibration of C-O-C. The lowest frequency peak at wavenumber $3450 \mathrm{~cm}^{-1}$ showed the strongest activity, which can be attributed to vibration of $\mathrm{O}(6) \mathrm{H}-\mathrm{O}(3)$ bond (Agarwal et al. 2011). Wavenumber $1639 \mathrm{~cm}^{-1}$ was related to absorbed water (Zhang et al. 2015). The acromion at $991 \mathrm{~cm}^{-1}$ showed the existence of arabia sugar side chain. The absorption at $897 \mathrm{~cm}^{-1}$ was the frequency vibration and ring frequency vibration of $\mathrm{C}-1$ group, which indicated that the main connections between hemicellulose units were $\beta$ glycosidic bonds (Bian et al. 2012).

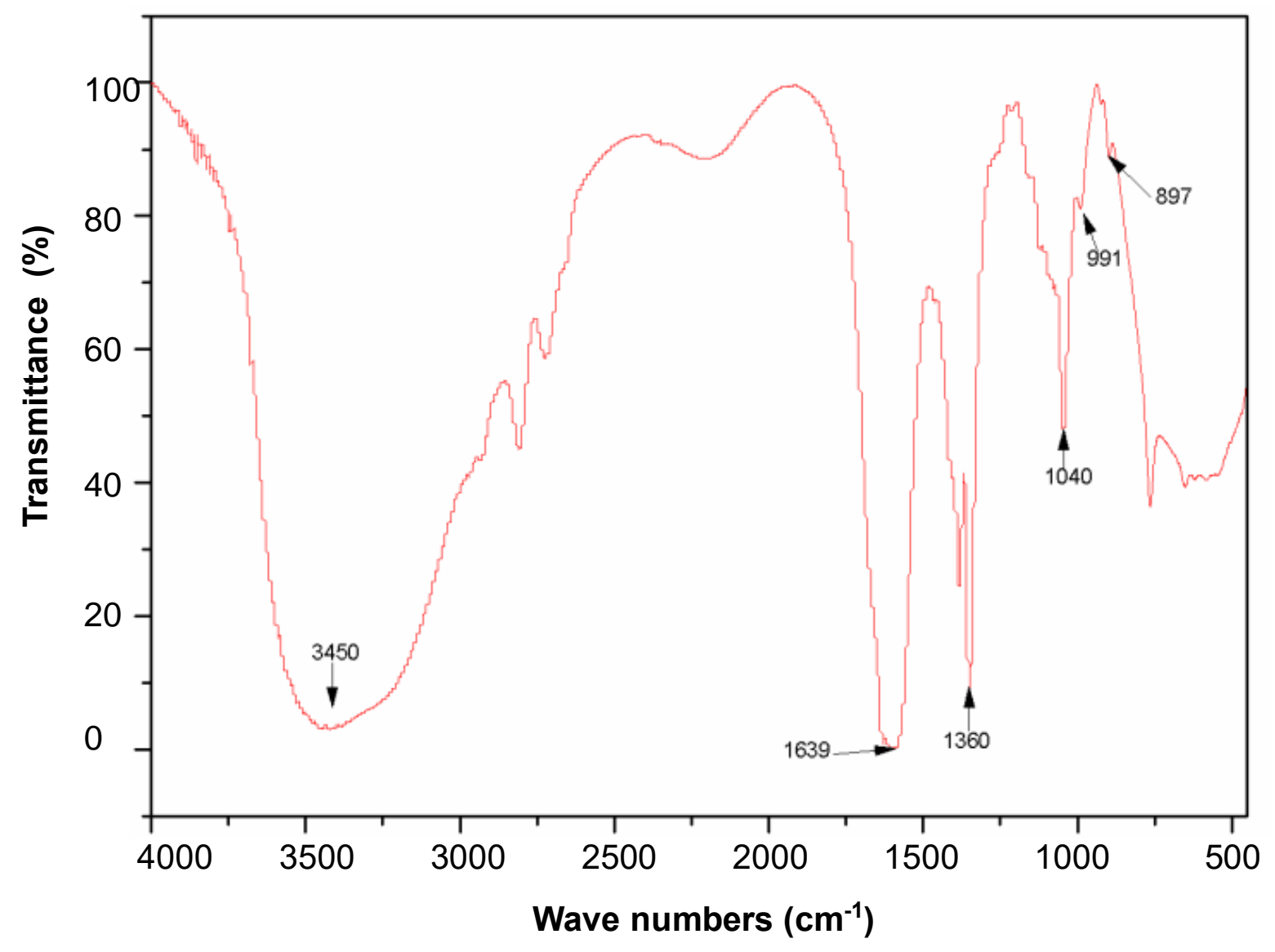

Fig. 3. FT-IR spectra of isolated hemicellulose from Spartina anglica

\section{PMP Derivatization and Separation Conditions}

Hemicellulose is mainly composed of xylan, which can be used to produce high value-added xylo-oligosaccharides (XOS) at industrial scale by enzymatic hydrolysis. However, rapid analysis of complex monosaccharides and oligosaccharides in hydrolysates 
of lignocellulosic biomass has remained a challenge. The commonly used methods for analysis of saccharides from lignocellulosic biomass are HPLC with a refractive index detector and GC analysis of alditol acetate derivatives (Krull and Inglett 1980; Slavin and Marlett 1983). The HPLC method is less sensitive for low concentrations of saccharides and gives a low resolution of oligo-saccharides.
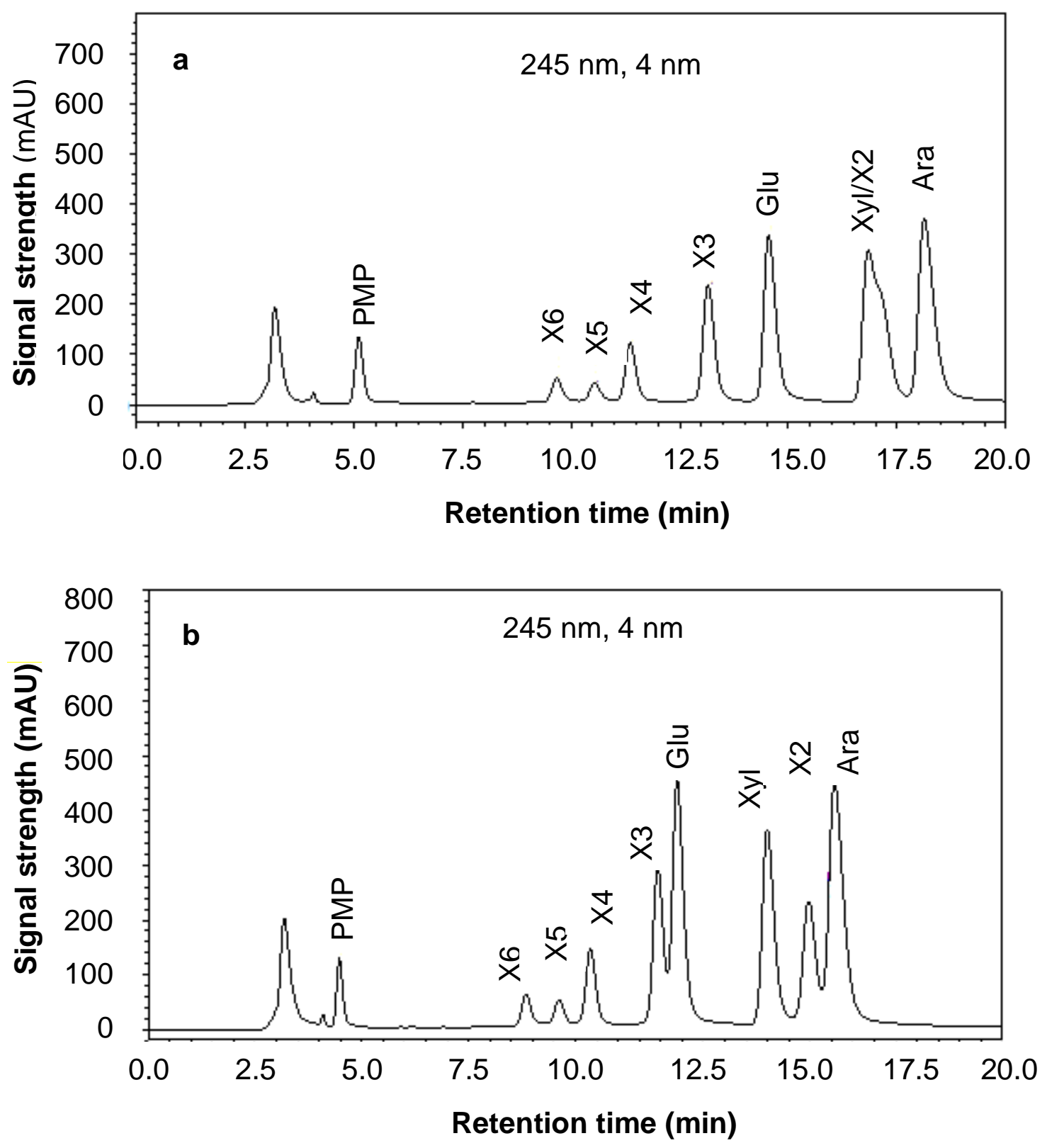

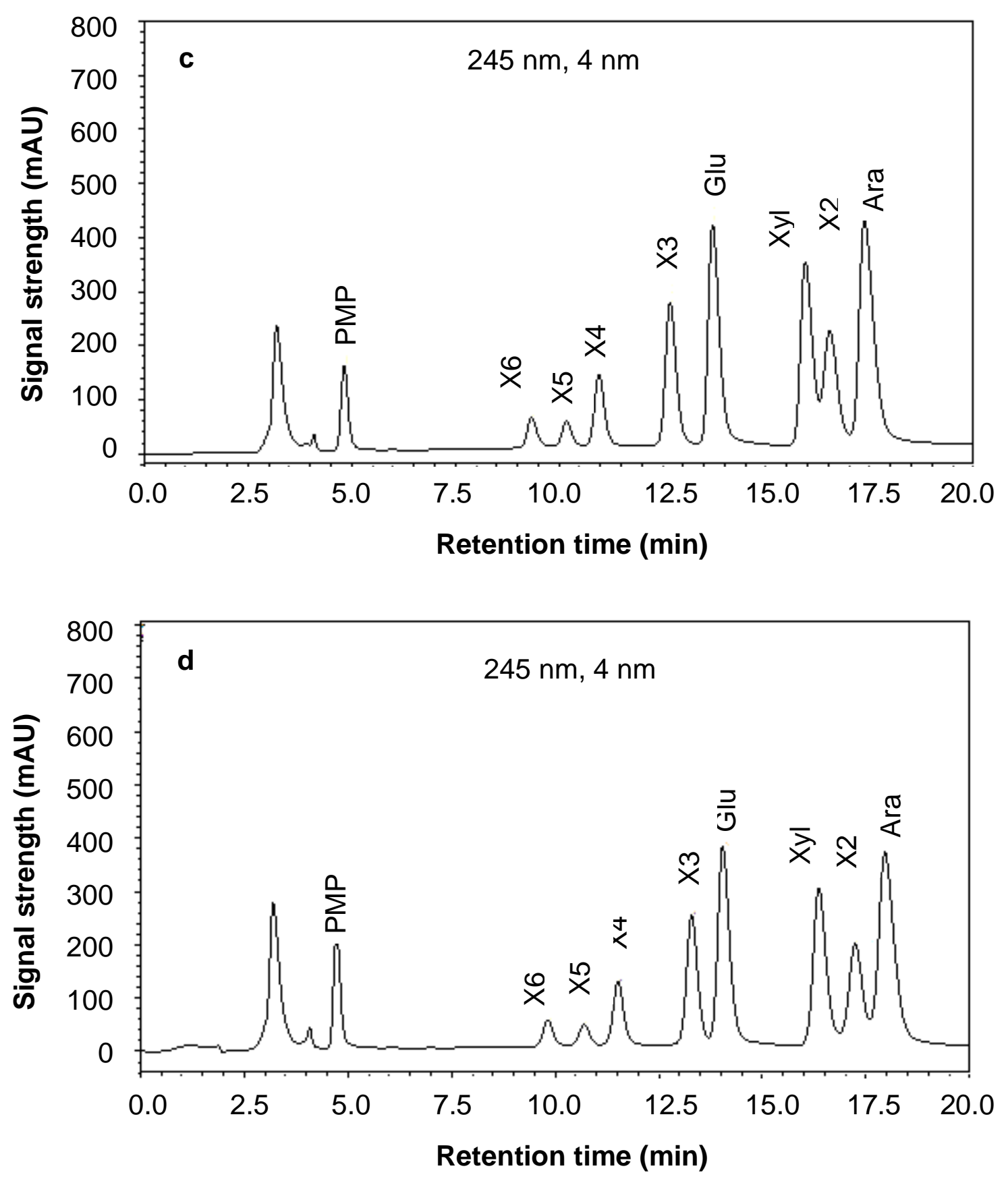

Fig. 4. Effect of mobile phase $\mathrm{pH}$ value on the separation of eight standard saccharides derivatives. a: $\mathrm{pH}$ 8.00; b: $\mathrm{pH} 8.10$; $\mathrm{c}$ pH 8.05; d: $\mathrm{pH} 8.06$

With consideration of the possible composition of hemicellulose, the standard monosaccharides solution (glucose, xylose, and arabinose) and xylooligosaccharides solution (xylobiose, xylotriose, xylotetraose, xylopentaose, xylohexaose) were prepared and derivatized with PMP. Linear regression was performed based on the measurement value calculated on the concentration of standard samples. Diluted standard solution was injected gradually to determine the minimum detection limit by calculating the concentration of standard solution when the noise-signal ratio was three. 
As shown in Fig. 4, PMP derivatized xylose and xylobiose could not be separated when the mobile phase $\mathrm{pH}$ was 8.00. Although baseline separation of both was achieved until $\mathrm{pH} 8.10$, xylotriose and glucose were not separated. However, according to the information shown in the chromatograms, it was possible to achieve separation within the $\mathrm{pH}$ range 8.00 to 8.10 . The eight kinds of PMP derivatized carbohydrate were separated completely at $\mathrm{pH}$ values up to 8.06. The retention time of PMP reagent was shorter than all the PMP derivatives, which will not interfere with the separation of PMP derivatives. This assay condition established the separation of monosaccharides (glucose, xylose, and arabinose) and xylooligosaccharides (xylobiose, xylotriose, xylotetraose, xylopentaose, xylohexaose), simplified the process steps, and improved efficiency.

\section{HPLC Analysis for Hemicellulose Hydrolysate}

The enzymolysis products of hemicellulose from Spartina anglica consist of monosaccharides and xylooligosaccharides. As shown in Fig. 5b, xylose, xylobiose, xylotriose, xylotetraose, xylopentaose, and xylohexaose were detected compared with the controlled trial (Fig. 5a). No glucose was detected, which suggested a high purity of hemicellulose. The full enzymolysis products were separated at $\mathrm{pH}$ 8.06. Xylobiose was the main xylooligosaccharide in the enzymolysis product.

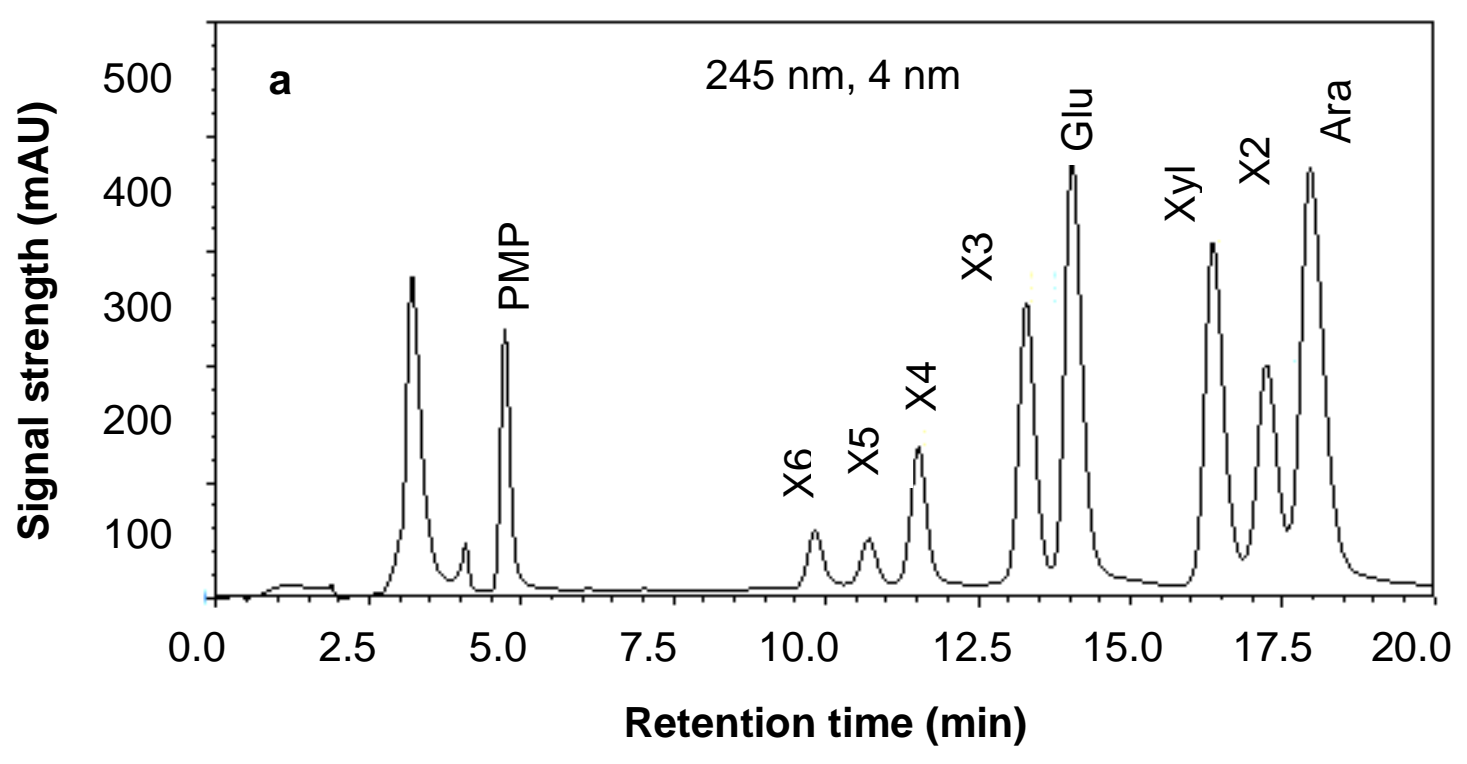




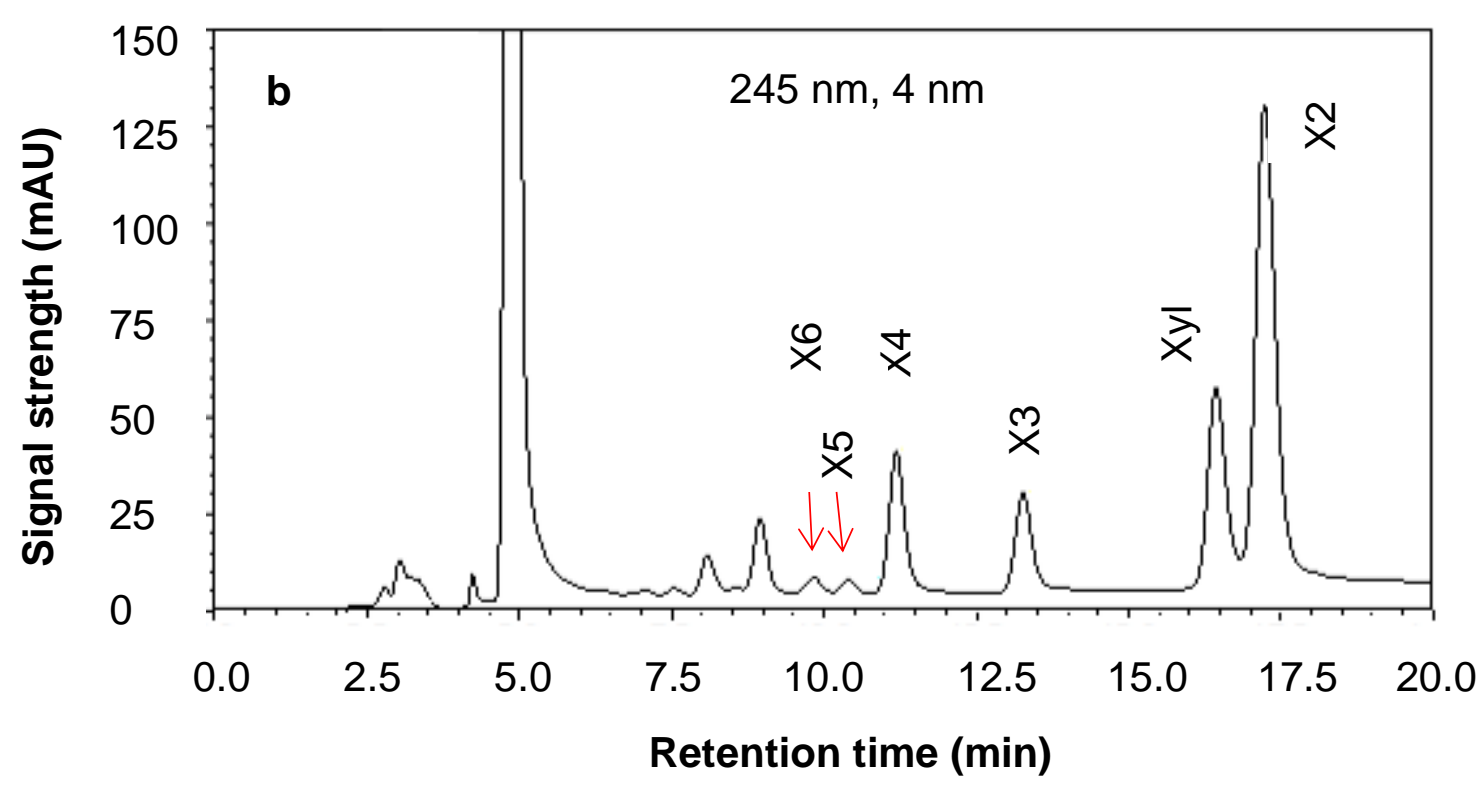

Fig. 5. Analysis of enzymatic hydrolysates of Spartina anglica hemicelluloses by HPLC. a: chromatogram of the standard $\mathrm{XOS}\left(\mathrm{X}_{2} \sim \mathrm{X}_{6}\right)$ derivatives; $\mathrm{b}$ : chromatogram of enzymatic hydrolysates of Spartina anglica hemicelluloses

\section{Regression Equation, Correlation Coefficient, the Minimum Detection Limit, and Precision Analysis using HPLC}

To test the accuracy of established method at short period, seven consecutive repeated experiments were performed, and the average relative standard deviations were calculated. Eight standard sugar regression equation, coefficient of determination $\left(\mathrm{R}^{2}\right)$, minimum detection limit (LOD), and average relative standard deviation (RSD) are shown in Table 1. Six kinds of standard sugar showed good linearity $\left(\mathrm{R}^{2}=0.9916\right.$ to 0.9995$)$ at 0.12 to $9.5 \mathrm{mM}$, sensitivity (LOD 1.60 to $2.27 \mu \mathrm{M}$ ), and precision (RSD of six kinds of standard sugar $<1.36 \%$ ). To evaluate the effect of PMP pre-column derivatization-HPLC method in natural production of xylooligosaccharides, this method was used to analyze the hemicellulose hydrolysis product of Spartina anglica using commercial xylanase. Xylotetraose, xylotriose, xylose, and xylobiose were separated and detected according to this method. Moreover, a small amount of xylopentaose and xylohexaose were detected at the same time (Fig. 5b). 
Table 1. Regression Equations, Coefficient $\left(\mathrm{R}^{2}\right)$, Limit of Detection $(L O D)$ and Average Relative Standard Deviations $(R S D)$ of the Proposed HPLC Method

\begin{tabular}{|c|c|c|c|c|}
\hline Saccharides & Regression equations & $\mathbf{R}^{2}$ & LOD $(\boldsymbol{\mu M})$ & $\begin{array}{c}\text { Average RSD } \\
(\%)\end{array}$ \\
\hline $\mathrm{X} 2$ & $\mathrm{y}=4.8 \times 10^{6} \mathrm{x}-140000$ & 0.9938 & 1.60 & 1.36 \\
\hline $\mathrm{X} 3$ & $\mathrm{y}=3.5 \times 10^{6} \mathrm{x}-19387$ & 0.9996 & 1.69 & 1.13 \\
\hline $\mathrm{X} 4$ & $\mathrm{y}=2.7 \times 10^{6} \mathrm{x}-63461$ & 0.9998 & 1.94 & 1.65 \\
\hline $\mathrm{X} 5$ & $\mathrm{y}=1.8 \times 10^{6} \mathrm{x}-66009$ & 0.9998 & 1.86 & 1.20 \\
\hline $\mathrm{X} 6$ & $\mathrm{y}=9.8 \times 10^{5} \mathrm{x}+22584$ & 0.9944 & 1.74 & 1.30 \\
\hline Glu & $\mathrm{y}=4.5 \times 10^{6} \mathrm{x}+760000$ & 0.9951 & 2.27 & 1.15 \\
\hline $\mathrm{Xyl}$ & $\mathrm{y}=7.1 \times 10^{6} \mathrm{x}+657379$ & 0.9990 & 1.66 & 1.18 \\
\hline Ara & $\mathrm{y}=9.3 \times 10^{6} \mathrm{x}+79040$ & 0.9925 & 1.68 & 1.14 \\
\hline
\end{tabular}

\section{CONCLUSIONS}

1. This study established a method to extract hemicellulose from Spartina anglica. The hemicellulose belongs to a kind of xylan and arabino-glycan. The purity of hemicellulose obtained by this method is at least $62.4 \%$, which could be used in the production of bio-based product.

2. A PMP-derivatization method was developed for simultaneous separation and analysis of monosaccharides and xylooligosaccharides. The method is suitable for use in the process monitoring of bioconversion. Three monosaccharides (glucose, xylose, and arabinose) and five xylooligosaccharides (xylobiose, xylotriose, xylotetraose, xylopentaose, xylohexaose) were simultaneously separated and analyzed, by using a C18 chromatographic column with UV detection wavelength at $245 \mathrm{~nm}$.

3. The PMP-derivatized method was successfully applied to analysis the enzymolysis products of hemicellulose from Spartina anglica. The enzymolysis products are consist of monosaccharide (xylose) and xylooligosaccharides (xylobiose, xylotriose, xylotetraose, xylopentaose, xylohexaose).

\section{ACKNOWLEDGMENTS}

This work was supported by Marine Biological Product Innovation Service Platform in Fujian Province [Grant numbers 2016-21] 


\section{REFERENCES CITED}

Agarwal, V., Huber, G. W., Jr, C. W., and Auerbach, S. M. (2011). "Simulating infrared spectra and hydrogen bonding in cellulose I $\beta$ at elevated temperatures," Journal of Chemical Physics 135(13), 134506.DOI: 10.1063/1.3646306

Arfelli, G., and Sartini, E. (2014). "Characterization of brewpub beer carbohydrates using high performance anion exchange chromatography coupled with pulsed amperometric detection," Food Chemistry 142(1), 152-158. DOI: 10.1016/j.foodchem.2013.07.008

Bian, J., Peng, F., Peng, X. P., Xu, F., Sun, R. C., and Kennedy, J. F. (2012). "Isolation of hemicelluloses from sugarcane bagasse at different temperatures: Structure and properties," Carbohydrate Polymers 88(2), 638-645.DOI: 10.1016/j.carbpol.2012.01.010

Brienzo, M., Carvalho, W., and Milagres, A. M. (2010). "Xylooligosaccharides production from alkali-pretreated sugarcane bagasse using xylanases from Thermoascus aurantiacus," Applied Biochemistry and Biotechnology 162(4), 1195. DOI: 10.1007/s12010-009-8892-5

Cao, C., Yang, Z., Han, L., Jiang, X., and Ji, G. (2015). "Study on in-situ analysis of cellulose, hemicelluloses and lignin distribution linked to tissue structure of crop stalk internodal transverse section based on FTIR microspectroscopic imaging," Cellulose 22(1), 139-149. DOI: 10.1007/s10570-014-0525-7

Honda, S., Akao, E., Suzuki, S., Okuda, M., Kakehi, K., and Nakamura, J. (1989). "High performance liquid chromatography of reducing carbohydrates as strongly ultraviolet-absorbing and electrochemically sensitive 1-phenyl-3-methyl-5pyrazolone derivatives," Analytical Chemistry 180, 351-357. DOI: 10.1016/00032697(89)90444-2

Lavarack, B. P., Griffin, G. J., and Rodman, D. (2002). "The acid hydrolysis of sugarcane bagasse hemicellulose to produce xylose, arabinose, glucose and other products," Biomass and Bioenergy 23(5), 367-380. DOI: 10.1016/S0961-9534(02)00066-1

Li, H., Long, C., Zhou, J., Liu, J., Wu, X., and Long, M. (2013). "Rapid analysis of mono-saccharides and oligo-saccharides in hydrolysates of lignocellulosic biomass HPLC," Biotechnology Letters 35(9), 1405. DOI: 10.1007/s10529-013-1224-4

Lin, Y., and Tanaka, S. (2006). "Ethanol fermentation from biomass resources: current state and prospects," Applied Microbiology and Biotechnology 69(6), 627-42. DOI: 10.1007/s00253-005-0229-x

Peng, F., Peng, P., Xu, F., Sun, R. C. (2012). "Fractional purification and bioconversion of hemicellulose," Biotechnology Advances 30(4), 879. DOI:

10.1016/j.biotechadv.2012.01.018

You, L., Yang, X. B., Yan, Z., Yun, R., Ying, Y., and Wang, Z. Z. (2009). "Separation and quantification of component monosaccharides of the tea polysaccharides from Gynostemma pentaphyllum by HPLC with indirect UV detection," Food Chemistry 112(3), 742-746. DOI: 10.1016/j.foodchem.2008.06.042

Zhang, L. Y., Xu, J., Zhang, L. H., Zhang, W. B., and Zhang Y. K. (2003). "Determination of 1-phenyl-3-methyl-5-pyrazolone-labeled carbohydrates by liquid chromatography and micellar electrokinetic chromatography," Journal Chromatography B 793, 159-165. DOI: 10.1016/S1570-0232(03)00373-8

Zhang, J., and Viikari, L. (2012b). "Xylo-oligosaccharides are competitive inhibitors of cellobiohydrolase I from Thermoascus aurantiacus," Bioresource 
Technology 117(4), 286-291. DOI: 10.1016/j.biortech.2012.04.072

Zhang, J., Tang, M., and Viikari, L. (2012a). "Xylans inhibit enzymatic hydrolysis of lignocellulosic materials by cellulases," Bioresource Technology 121(121), 8-12. DOI: 10.1016/j.biortech.2012.07.010

Zhang, P., Dong, S. J., Ma, H. H., Zhang, B. X., Wang, Y. F., and Hu, X. M. (2015). "Fractionation of corn stover into cellulose, hemicellulose and lignin using a series of ionic liquids," Industrial Crops and Products 76, 688-696. DOI: 10.1016/j.indcrop.2015.07.037

Article submitted: February 3, 2020; Peer review completed: April 18, 2020; Revised version received: July 30, 2020; Accepted: July 31, 2020; Published: August 19, 2020. DOI: $10.15376 /$ biores.15.4.7628-7639 